\title{
Parallel Piezoelectric Shunt Damping of Rotationally Periodic Structures
}

\author{
Bilal Mokrani, ${ }^{1}$ Renaud Bastaits, ${ }^{1}$ Mihaita Horodinca, ${ }^{2}$ Iulian Romanescu, ${ }^{2}$ Ioanica Burda, ${ }^{3}$ \\ Régis Viguié, ${ }^{4}$ and André Preumont ${ }^{1}$
}

${ }^{1}$ Active Structures Laboratory, Université Libre de Bruxelles, 50 Avenue Franklin Roosevelt, 1050 Brussels, Belgium

${ }^{2}$ Technical University "Gheorghe Asachi”, 67 Boulevard Professor Dimitrie Mangeron, 700050 Iasi, Romania

${ }^{3}$ Babes Bolyai University, 1 Mihail Kogalniceanu Street, 400084 Cluj-Napoca, Romania

${ }^{4}$ Groupe SAFRAN, Techspace Aero, 121 Route de Liers, 4041 Liège, Belgium

Correspondence should be addressed to Bilal Mokrani; bilal.mokrani@ulb.ac.be

Received 15 December 2014; Revised 24 March 2015; Accepted 24 March 2015

Academic Editor: João P. Davim

Copyright (C) 2015 Bilal Mokrani et al. This is an open access article distributed under the Creative Commons Attribution License, which permits unrestricted use, distribution, and reproduction in any medium, provided the original work is properly cited.

\begin{abstract}
This paper considers the RL shunt damping of rotationally periodic structures with an array of regularly spaced piezoelectric patches. The technique is targeted to the damping of a specific mode with $n$ nodal diameters. For this particular case, one can take advantage of the shape of the targeted mode to organize the piezoelectric patches as a modal filter (in parallel loops) which reduces the demand on the inductors of the tuned inductive shunt. In the case of a perfectly rotationally periodic structure, it is possible to organize $4 n$ piezoelectric transducers (PZT patches) in two parallel loops of $2 n$ patches each. In this way, the demand on the inductors is reduced by $4 n^{2}$ as compared to independent loops, which may allow a fully passive integration of the RL shunt in a turbomachinery application. The method is first illustrated experimentally on a circular plate; it is then applied to a prototype of an industrial bladed drum. The influence of blade mistuning is investigated.
\end{abstract}

\section{Introduction}

New materials and new fabrication techniques in turbomachinery (e.g., blisks) lead to structures with extremely low damping which may be responsible for severe vibrations and possible high-cycle fatigue problems. The damping may be increased in various ways: blade friction damping [1], friction ring damper [2], viscoelastic damping treatment, or piezoelectric shunt $[3,4]$, to name only a few.

The use of piezoelectric transducers for damping structures has been known for a long time [5-8]; the piezoelectric transducer is used to convert the mechanical energy into electrical energy, which is dissipated in an electrical network. The R-shunt involves only a network of resistors; it has limited performances, but it is simple and robust. The RL shunt involves a set of resistors and inductors and the electrical network is tuned on the targeted mode. Damping simultaneously several modes is possible [9]. The performances are superior to the R-shunt, but the electrical network must be tuned accurately on the frequency of the targeted mode(s); it does not damp the other modes and, for the targeted ones, it is very sensitive to the variations of the natural frequencies. For most practical applications, the value of the inductance $L$ required to achieve electrical tuning, $(L C)^{-1 / 2} \simeq \omega_{i}$, is very large, which necessitates using synthetic inductors (electronic circuits called gyrator) [10]. Active components such as synthetic inductors are difficult to implement in rotating machines. The problems of the passive RL shunt may be overcome by using a switched shunt [11]; it does not require to be precisely tuned, nor a large inductor, but the switching synchronization is an issue, particularly when several vibration modes are involved, and, once again, active circuits make this solution impractical in rotating machines.

In turbomachines, the blades are subjected to excitation frequencies which are multiple of the rotation speed (depending on the number of stator vanes). The normal modes of rotationally periodic structures consist of harmonically varying displacements in the circumferential direction which makes them very similar to axisymmetric structures [12]. Depending on the geometry of the stator part and the 


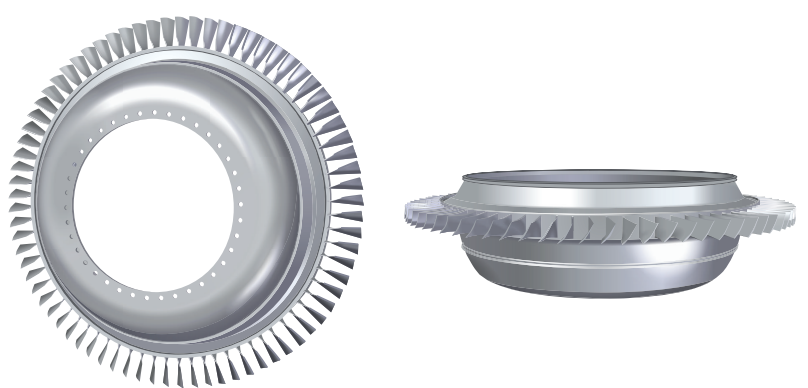

FIGURE 1: Bladed drum considered in this study.

operating speed of the rotor, it is possible to identify the critical mode with a number $n$ of nodal diameters, which is likely to be excited and must be targeted for damping.

This paper presents a method for damping a particular mode with $n$ nodal diameters: it consists of organizing the piezoelectric transducers in parallel loops grouping several of them to a single inductor. A set of $4 n$ piezoelectric transducers (PZT patches) may be arranged in two parallel loops of $2 n$ patches each; this allows reducing the demand on the inductors by $4 n^{2}$ as compared to independent inductive loops, while providing the same amount of damping on the targeted mode with $n$ diameters. This offers the possibility of a purely passive integration of the piezoelectric RL shunt damping for turbomachinery structures. The method is illustrated on a circular plate and on a bladed drum with 76 blades (Figure 1). The effect of blade mistuning on performance is investigated.

\section{Rotationally Periodic Structures}

A bladed disk or drum equipped with $N$ blades is said to have cyclic symmetry, with an interblade phase angle of $2 \pi / N$. It is possible to analyze the complete structure by considering only one substructure $[13,14]$. Considering two points at the same relative position in two adjacent sectors, the free vibration modes of cyclic structures are harmonic in the circumferential direction, which leads to nodal lines across the mode shapes called nodal diameters. Except for the modes with 0 and $N / 2$ nodal diameters for an even number $N$ of blades, the modes occur by pairs (sine and cosine modes) with the same natural frequency.

The natural frequencies are usually plotted as a function of the number $n$ of nodal diameters as in Figure 2 (the plot depends on the rotation speed); the maximum number of nodal diameters is $N / 2$ if $N$ is even or $(N-1) / 2$ if $N$ is odd. The frequencies of the drum-dominated modes tend to increase rapidly with the number of nodal diameters while the families of the blade-dominated modes appear as horizontal lines: first flexural mode denoted by $1 \mathrm{~F}$ (corresponding to the first bending mode of a cantilevered blade), first torsion mode 1T, second flexural mode $2 \mathrm{~F}$, and so forth. For these modes, the strain energy is dominantly in the blades, although the drum accounts also for a small amount of it. The modes in the veering region where blade-dominated modes meet the drum-dominated modes tend to have significant

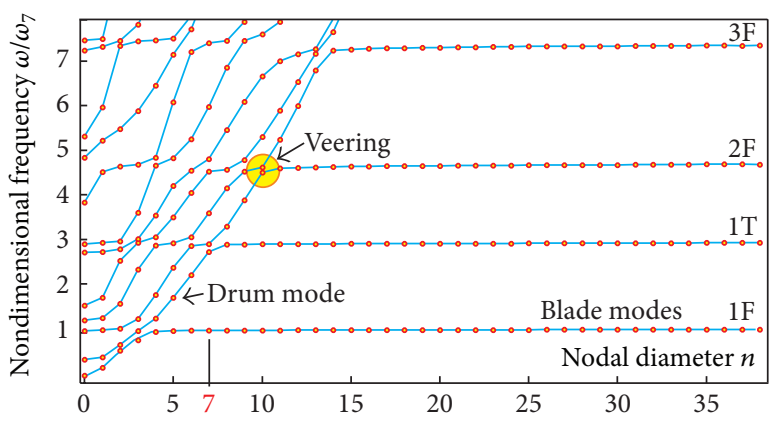

Figure 2: Natural frequencies (normalized to $\omega_{7}$ ) versus nodal diameters $n$ for the bladed drum of Figure 1 (for a given rotation speed $\Omega$ ).

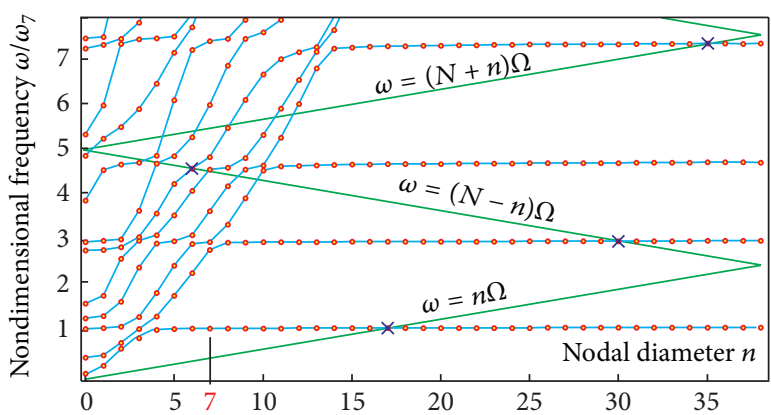

FIGURE 3: Resonance condition to a rotating force. Zig-zag lines superimposed on the natural frequencies versus nodal diameters plot of the bladed drum with $N=76$ blades. The resonance conditions are indicated by $x$.

deflections (and strain energy) in both the blades and the drum [15].

The forced response of periodic structures to a rotating point force has been analyzed by Wildheim [12]. For a rotationally symmetric structure excited by a rotating force (at $\Omega$ ), the resonance of the mode with $n$ nodal diameters is obtained when the natural frequency satisfies $\omega_{n}=n \Omega$. For a rotationally periodic structure with $N$ substructures, additional resonance possibilities exist, given by

$$
\omega_{n}=(k N \pm n) \Omega,
$$

where $k=1,2, \ldots$. For a given rotation speed, these resonance conditions give a zig-zag line in the natural frequency versus nodal diameters diagram, which can be superimposed on the diagram of natural frequencies (for the same $\Omega$ ), leading to Figure 3. Notice that the zig-zag line corresponds to a given rotation speed; if $\Omega$ increases, the line expands like an accordion along the frequency axis. All the intersections between the two sets of lines, provided they occur at an integer value of $n$, correspond to a resonance condition, indicated by $\times$. If the crossing occurs in the ascending part of the zig-zag line $\left[\omega_{n}=(k N+n) \Omega\right]$, the resonant response consists of a forward rotating wave in the direction of the excitation; if the crossing occurs in the descending part of the zig-zag line $\left[\omega_{n}=(k N-n) \Omega\right]$, the resonant response consists of a backward traveling wave. 


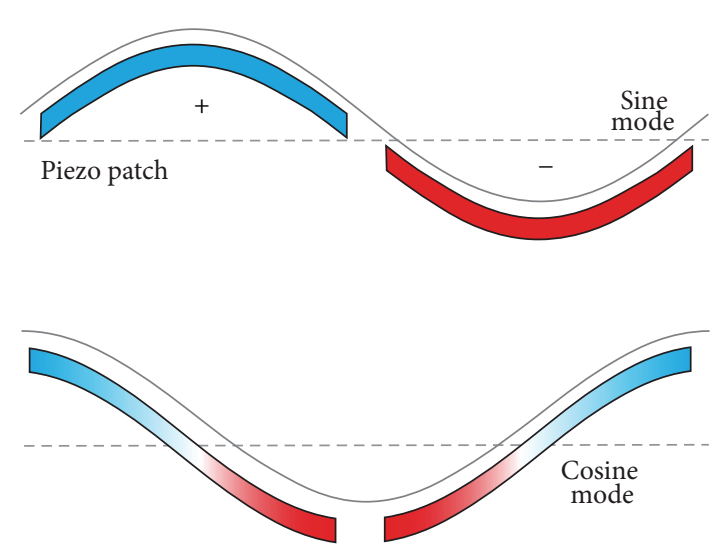

(a)
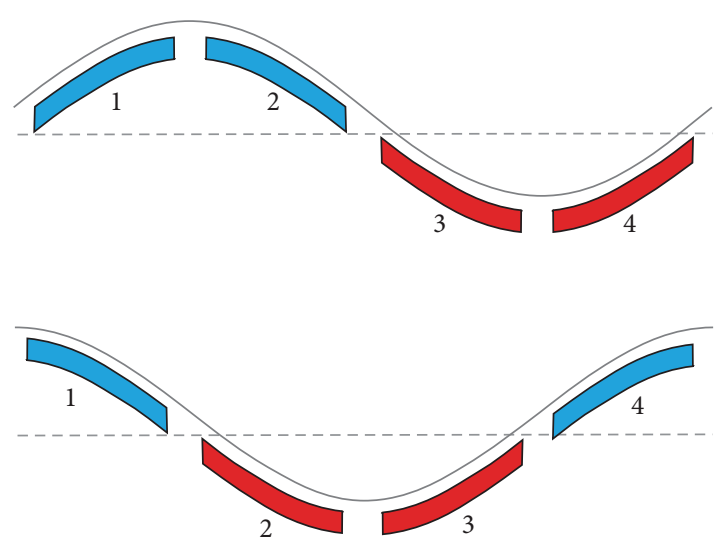

(b)

FIGURE 4: Curvature of piezoelectric patches within one nodal diameter of the sine and cosine modes of a mode with $n$ diameters, for two configurations of the PZT patches. (a) Two patches per nodal diameter. (b) Four patches per nodal diameter.

In turbomachinery, the excitation itself is periodically distributed (engine order excitation). For an excitation consisting of $s$ rotating point forces, the resonance condition is more elaborate and involves the double equality [12]

$$
\omega_{n}=(k N+n) \Omega=h_{1} s \Omega
$$

or

$$
\omega_{n}=(k N-n) \Omega=h_{2} s \Omega,
$$

where $h_{1}$ and $h_{2}$ are any nonzero integers. The second condition reduces drastically the points on the zig-zag line which qualify for a resonance.

Without elaborating further on the determination of the resonance, the point we want to make is that not all the modes are dangerous and, depending on the excitation order (e.g., air flow shaped by the geometry of stator parts) and the operating speed of the rotor, it is possible to identify the critical mode with a number $n$ of nodal diameters which is likely to be excited and must be targeted for damping. The present paper addresses the damping of a specific mode with $n$ nodal diameters.

\section{RL Shunt Damping}

3.1. Independent Loops. When many PZT patches are available, several architectures are possible. We first examine the damping with independent RL loops. If a mode with natural frequency $\omega_{i}$ is targeted, the inductance of the RL shunt should be selected according to

$$
L=\frac{1}{C \omega_{i}^{2}},
$$

where $C$ is the electrical capacitance of one PZT patch. The performance of the RL shunt depends critically on the tuning of the inductor; it is less sensitive to the value of the resistance $R$; the value of $R$ which maximizes the damping of the poles corresponding to the targeted mode (this value of $R$ brings the poles of the electrical circuit to the same location [16, p.180]) is given by

$$
R=\frac{2 K_{i}}{C \omega_{i}},
$$

where $K_{i}$ is the total effective electromechanical coupling factor of mode $i$, the sum of the effective electromechanical coupling factors of the $p$ transducers:

$$
K_{i}^{2}=\sum_{j=1}^{p} K_{i}^{2},
$$

where ${ }^{j} K_{i}{ }^{2}$ is the effective electromechanical coupling factor of transducer $j$ for mode $i$ (observe that although the loops are independent, the resistance depends on the sum of the effective electromechanical coupling factors of all the patches $[17$, ch.2]).

If, as often happens in bladed structures, the natural frequencies of a set of $N$ modes are very close to each other, within 2 or $3 \%$, the tuning of the RL shunt may again be based on (4), using the average frequency $\bar{\omega}=\sum \omega_{i} / N$. The tuning of the resistor requires the knowledge of the electromechanical coupling factors, which are different for modes with different mode shapes; however, the effectiveness of the RL shunt is less sensitive to the tuning of the resistor and it is sufficient to select the resistance according to (5) with the average value of $K_{i}^{2}$ over the entire set of $N$ modes:

$$
K_{i}^{2}=\frac{1}{N} \sum_{i=1}^{N} \sum_{j=1}^{p}{ }^{j} K_{i}^{2} .
$$

All the $p$ RL shunt circuits have exactly the same tuning.

3.2. Parallel Loops. When a particular mode with $n$ nodal diameters is targeted, one may take advantage of the mode shape in the following way: Figure 4 shows the sine and cosine mode shapes in the circumferential direction along an angle 

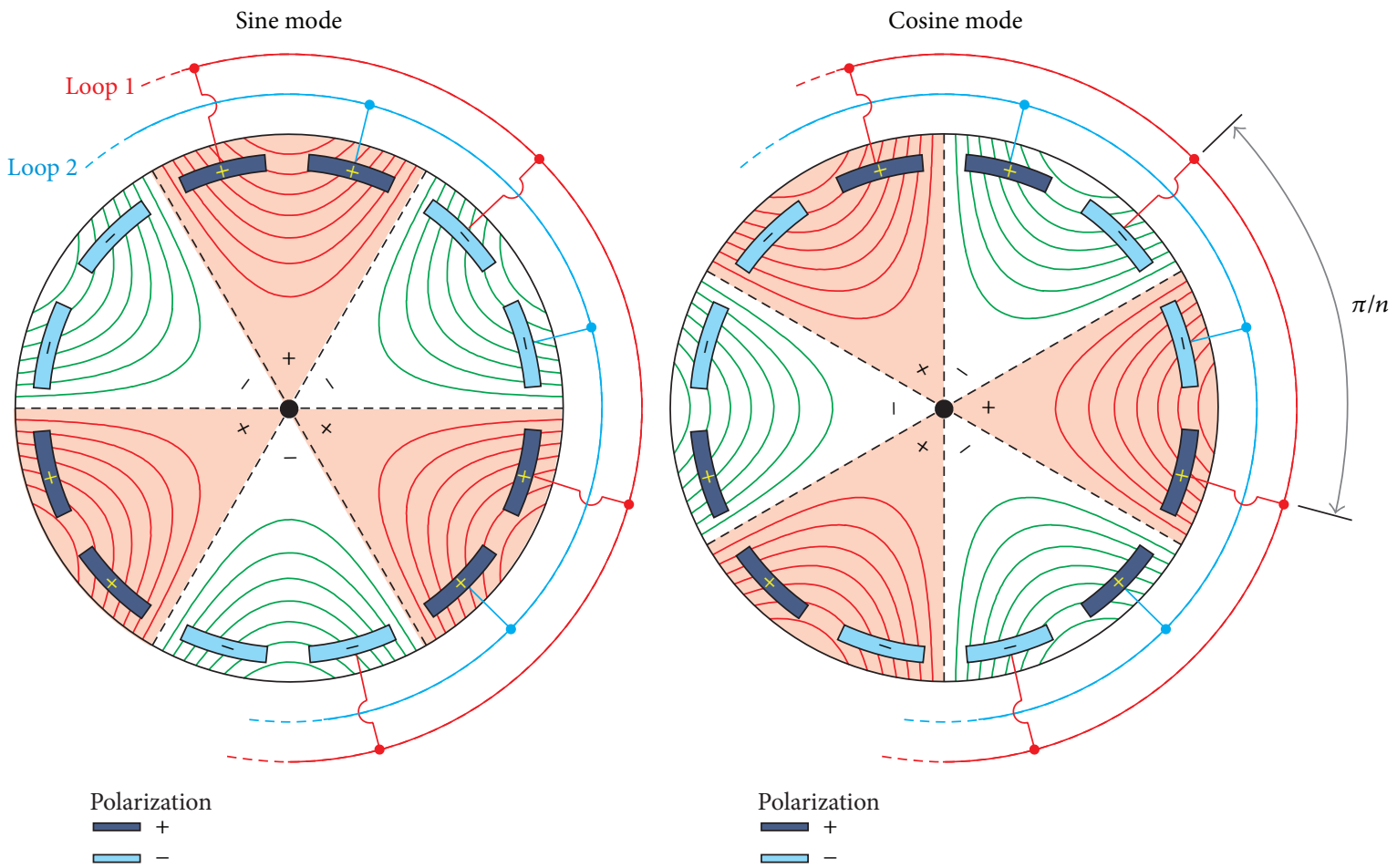

FIGURE 5: Parallel loop implementation of the shunt damping: electrical connections and polarization diagram illustrated for a mode with $n=3$ nodal diameters of natural frequency $\omega_{i}$. Sine mode and cosine mode. The color on the disk indicates the sign of the curvature and the color of the patches indicates the polarization.

$2 \pi / n$ corresponding to one nodal diameter; the antinodes of the cosine mode correspond to the nodes of the sine modes and vice versa; if the size of the electrodes on the PZT patches is such that there are only 2 independent electrodes per nodal diameter (Figure 4(a)), one electrode covers entirely one lobe of the mode shape and the curvature cannot be kept of the same sign over the entire electrode simultaneously for the sine and the cosine modes. In Figure 4(a), the curvature of the patches covering the cosine mode changes sign and, as a result, the electric charges produced on one half of the electrode are canceled by those produced by the opposite curvature on the other half of the electrode. Overall, no electric charge is produced. By contrast (Figure 4(b)), if the electrode layout is such that there are 4 independent electrodes per diameter (or a multiple thereof), the curvature keeps always the same sign over the electrodes. Besides, the curvature of the patches numbered 1 and 3 on Figure 4(b) has opposite signs for both the sine and cosine modes and similarly for the patches numbered 2 and 4 . This suggests that if the patches number 1 and number 3 are mounted with inverted polarization, they can be mounted in parallel and act together and similarly for patches number 2 and number 4 .

The foregoing observation suggests the implementation illustrated in Figure 5 for a mode with $n=3$ nodal diameters of a disk clamped at the center: the $4 n$ patches (or a multiple thereof) are mounted by pairs with identical polarization (note that it is not necessary for the patches to be physically separated, provided that the electrodes are independent), with the next pair having inverted polarization. The electrodes are then connected in two independent loops of $2 n$ each connecting electrodes with opposite polarization separated by an angle $\pi / n$. Finally, there are only two electrical circuits having an electrical capacitance of $2 n C$ each; the inductance (tuned on the frequency $\omega_{i}$ ) of one loop becomes

$$
L=\frac{1}{2 n C \omega_{i}^{2}} .
$$

Thus, the overall inductance requirement is reduced by $4 n^{2}$ with respect to the independent loops, which is appreciable if $n$ is large. The optimum resistance is given by

$$
R=\frac{2 K_{i}}{2 n C \omega_{i}},
$$

where the effective electromechanical coupling factor is in this case

$$
K_{i}^{2}=\frac{1}{2} \sum_{j=1}^{p}{ }^{j} K_{i}^{2}=2 n \cdot{ }^{j} K_{i}^{2},
$$

where ${ }^{j} K_{i}^{2}$ refers to a single patch (identical for all).

3.3. Experiment on a Circular Plate. A comparison of the independent loops and parallel loops shunt damping has been performed on an aluminum disk of $300 \mathrm{~mm}$ diameter and $2 \mathrm{~mm}$ thickness (Figure 6); it is equipped with $12 \mathrm{PZT}$ rectangular patches of $40 \mathrm{~mm} \times 10 \mathrm{~mm} \times 200 \mu \mathrm{m}$ (PIC255 from P-I). The patches are glued in such a way that the polarization matches that of Figure 5. A set of 12 synthetic 


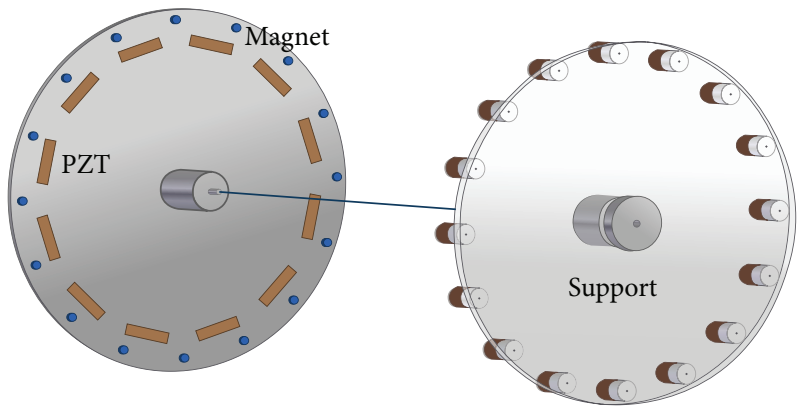

(a)

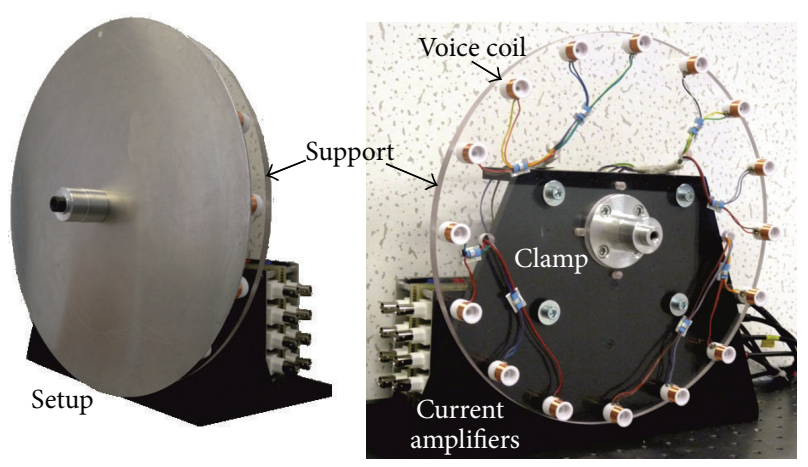

(b)

FIGURE 6: Circular plate clamped at the center equipped with $12 \mathrm{PZT}$ patches. (a) CAD view of the setup, (b) experimental setup including 16 noncontact voice coil actuators.

inductors provide 12 independent RL channels. A set of 16 noncontact voice coil actuators interacting with small permanent magnets attached to the disk (each magnet weighs $1.9 \mathrm{~g}$ ) provide 16 independent point force excitations which may be used to excite specific modes (appropriate excitation), including rotating ones. The voice coils are connected to an array of 16 current amplifiers which are controlled by a DSP board. More details about the experimental setup are available in [17].

The experimental comparison between the independent RL shunt (12 independent loops) and the parallel shunt (2 loops involving 6 PZT patches each) is shown in Figure 7. In both cases, synthetic inductors are used. The figure compares the FRF between a point force $F$ and the plate velocity $\dot{x}$ (obtained without contact with a laser vibrometer) in the vicinity of the first mode with $n=3$ diameters, at three different points: Figure 7(a) corresponds to the point where the response of the sine mode is maximized; Figure 7(b) corresponds to the point where the response of the cosine mode is maximized; Figure 7(c) is in the midway, where both modes contribute to the response. The two strategies exhibit similar performances.

\section{RL Shunt Damping of a Bladed Drum}

The monobloc bladed drum considered in this study is represented in Figure 8. Since it is fabricated in a single piece, the natural damping is extremely small, of the order of $\xi \simeq 10^{-4}$. Right from the beginning of this project, placing the PZT patches on the blades was ruled out, to avoid any interference with the aerodynamics. As a result, 28 PZT patches working in $d_{31}$ mode have been glued to cover almost completely the inner side of the blade support rim (Figure 8). Calculations and preliminary tests on a bladed rail showed that this configuration provides enough authority for damping vibration of the blade-dominated modes [18]. A model has been developed with Mindlin shell elements in the finite element software SAMCEF; the model includes specific piezoelectric elements for 28 PZT patches $[19,20]$; a state space model involving 150 modes has been created in MATLAB; the inputs of the model are 76 point forces $f_{i}$ acting on the tip blades and 28 voltages $V_{i}$ controlling the PZT patches. The output of the model includes the tip blade velocities $\dot{x}_{i}$ and the electrical charges on the PZT patches. The MATLAB model allows us to implement the various RL shunt strategies and to simulate various types of excitations with rotating forces.

4.1. Numerical Comparison. The two damping strategies discussed above have been compared on the model of the bladed drum; since there are 28 PZT patches, the shunt damping with parallel loops may be implemented for the mode with $n=$ 7 nodal diameters. The implementation with independent loops is targeted at all the modes of the $1 \mathrm{~F}$ family, while the implementation with parallel loops will target the mode $1 \mathrm{~F} 7$ (mode with 7 diameters of the $1 \mathrm{~F}$ family). If $\eta(t)$ is a bandlimited white noise in a frequency band $\left[\omega_{1}, \omega_{2}\right]$ including all the $1 \mathrm{~F}$ modes, the distributed forcing function

$$
F(\theta, t)=\eta(t) \sum_{j=0}^{N / 2} \cos j \theta
$$

excites all the $1 \mathrm{~F}$ modes (it includes a contribution which is appropriate for all the modes). Since the model assumes $N=$ 76 regularly spaced discrete actuators, this forcing function is discretized according to

$$
f_{k}(t)=F\left(\frac{2 \pi k}{N}, t\right)=\eta(t) \sum_{j=0}^{N / 2} \cos \left(\frac{j 2 \pi k}{N}\right) .
$$

Figure 9 compares the average response of all the blades to the foregoing excitation, with and without independent loop shunt (all the inductors of the RL loops are tuned on the natural frequency of the 1 F7 mode, $\omega_{7}$ ). Figure $9(\mathrm{a})$ is the normalized FRF $T(\omega)$ in the frequency band in the vicinity of the 1F modes and Figure 7(b) shows the cumulative RMS response defined as

$$
\sigma(\omega)=\left[\int_{\omega_{1}}^{\omega}|T(\nu)|^{2} d v\right]^{1 / 2}
$$

One can observe that the RL shunt with independent loops affects most of the $1 \mathrm{~F}$ modes (not all, because there are only 28 patches); this is due to the close values of the natural frequencies of all the modes of the $1 \mathrm{~F}$ family. For this example, each of the 28 electrical circuits would require an inductor of $0.7 \mathrm{H}$, which is not feasible with passive components. 


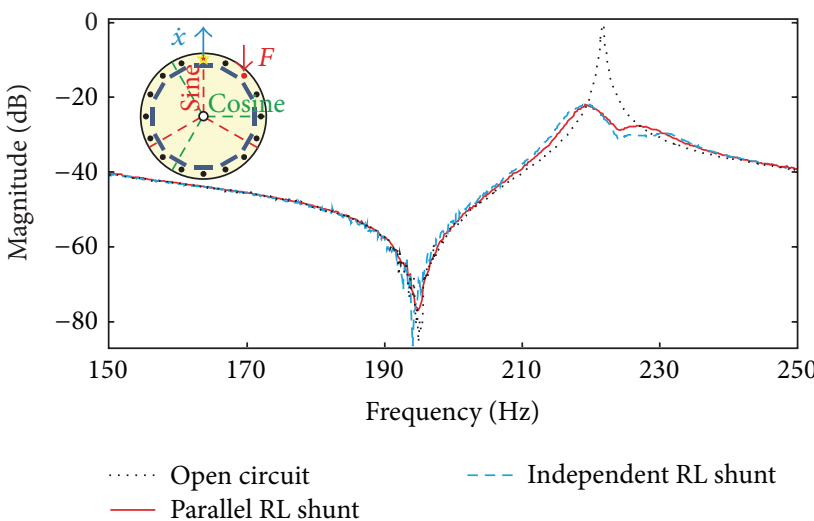

(a)

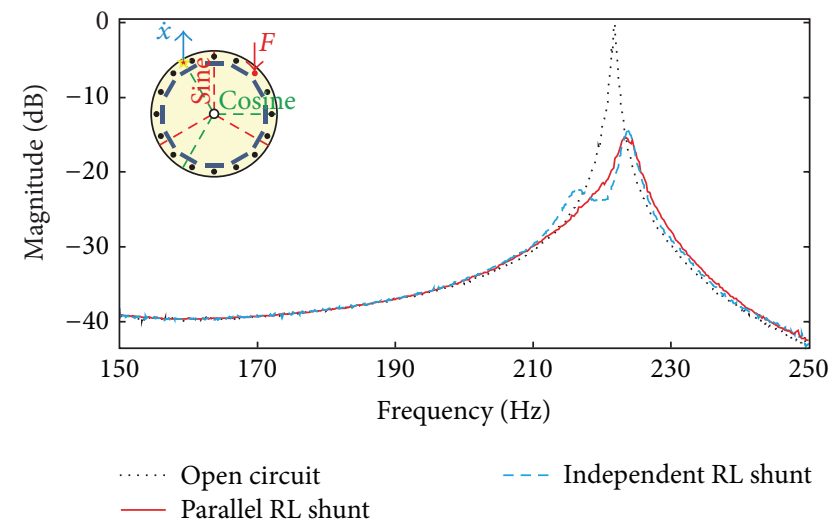

(b)

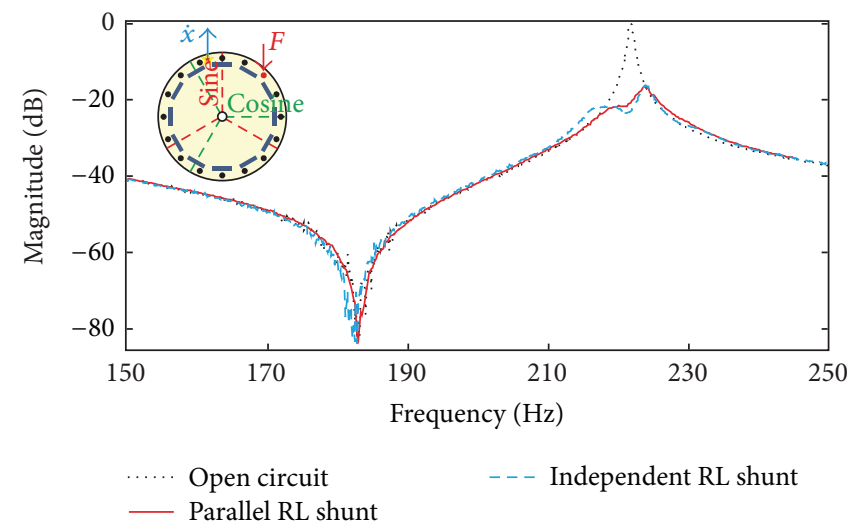

(c)

FIGURE 7: Disk experiment: comparison between the independent shunt and parallel shunt for the first mode with $n=3$ nodal diameters. The dotted lines correspond to the original system, with a natural damping of $0.5 \%$. (a) $\dot{x}$ is measured at the point where the sine mode is maximized. (b) $\dot{x}$ is measured at the point where the cosine mode is maximized. (c) $\dot{x}$ is measured in the midway, where both modes contribute to the response.

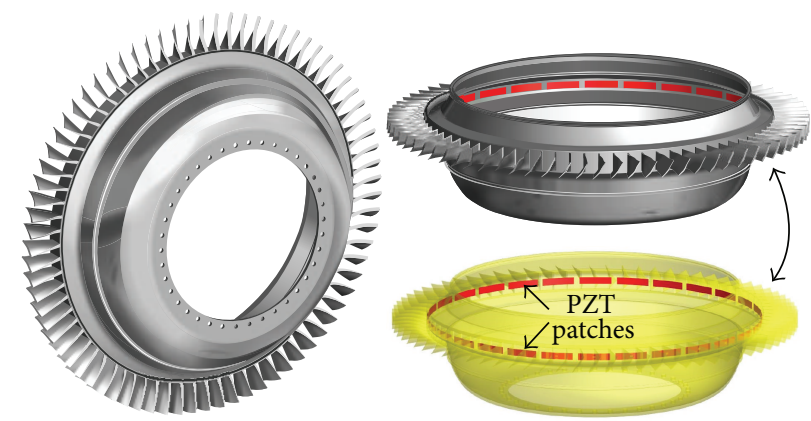

FIGURE 8: Bladed drum used in this study. Layout of the PZT patches on the inner side of the blade support rim.

The damping with parallel loops is considered in Figure 10. In this case, the 28 PZT patches are connected in two independent electrical loops of 14 patches each, with a capacitance of $14 C$; this reduces the requirement on the inductor to only 2 inductors of about $50 \mathrm{mH}$ (instead of 28 inductors of $0.7 \mathrm{H}$ in the case of independent loops). Figure 10 shows the FRF between the forcing function and the tip velocity of one blade in a very narrow frequency band in the vicinity of $\omega_{7}$; the FRF is represented for three conditions: open circuit, independent loops, and parallel loops. The parallel loops curve is almost superimposed on the curve for open circuit, except for the targeted mode 1F7. The effect of the parallel loops shunt on mode $1 \mathrm{~F} 7$ is nearly the same as that of the independent loops shunt, although the latter affects all the modes in the vicinity.

\section{Experiment}

5.1. Experimental Setup. The experimental setup is shown in Figure 11; it consists of a one stage, single piece bladed 


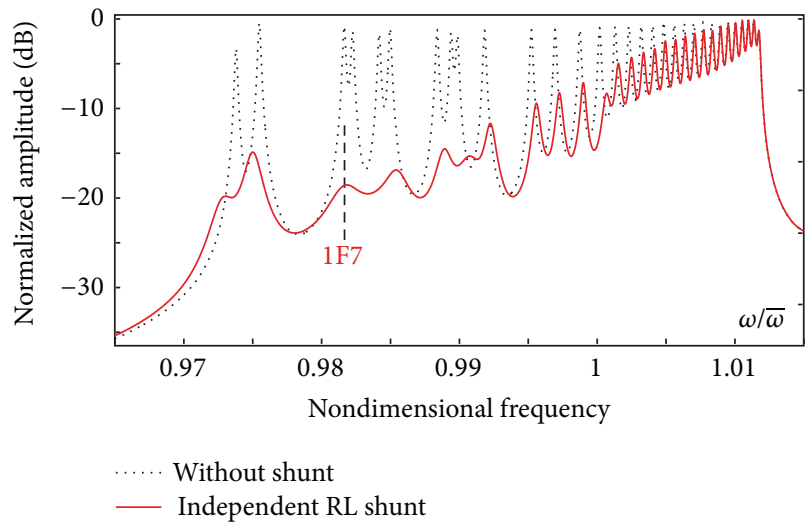

(a)

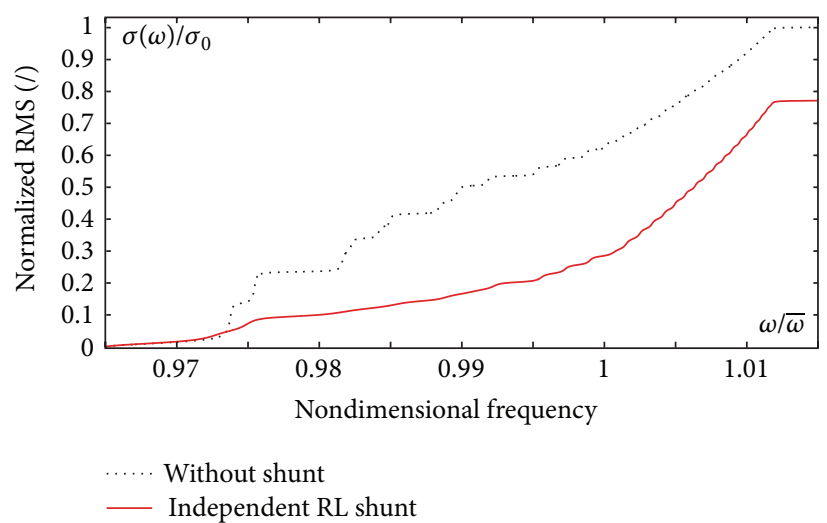

(b)

Figure 9: Simulation response of one blade with independent RL loops. (a) Normalized FRF $T(\omega)=|\dot{x} / \eta|$ between the forcing function and the velocity measured at the blade tip, (b) cumulative RMS of $\sigma(\omega)$.

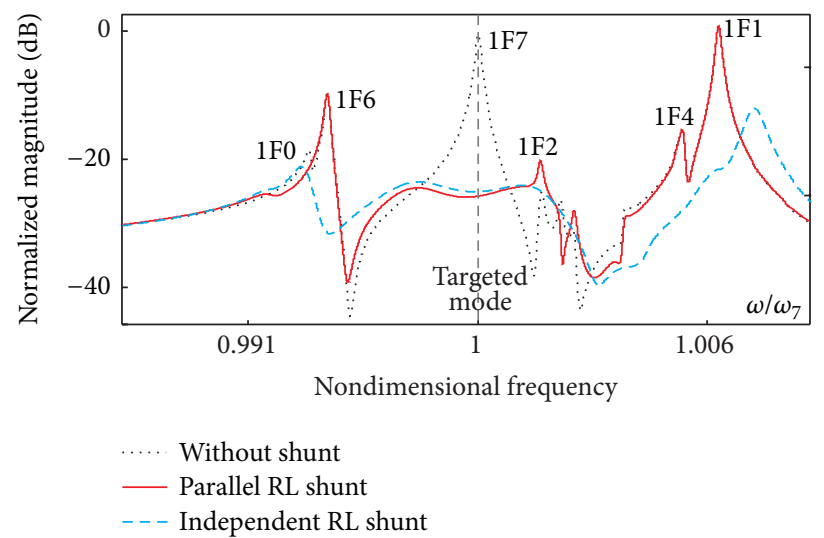

FIGURE 10: Simulation. Normalized FRF $T(\omega)=|\dot{x} / \eta|$ between the forcing function and the velocity of the tip of one blade in the vicinity of $\omega_{7}$; effect of the RL shunt tuned on mode $1 F 7$ with both configurations: independent and parallel shunt.

stainless steel drum with 76 blades welded on the outer rim of the drum. The natural damping has been measured in the range of $\xi \simeq 10^{-4}$ (this small value justifies the investigation of damping enhancement techniques). The inner side of the drum opposing the foot of the blades is covered by $28 \mathrm{PZT}$ patches of rectangular shape $(40 \mathrm{~mm} \times 10 \mathrm{~mm} \times 200 \mu \mathrm{m}$; PIPIC255) operating in the $d_{31}$ mode. The PZT patches have all been glued with the same polarity and do not follow the layout of Figure 5 (this was done very early in the project, before the development of the damping strategy based on parallel loops); as a result, the parallel loop implementation requires four independent loops instead of two. A set of 28 synthetic inductors (based on Antoniou's circuit [21]) have been built to provide 28 independent tunable RL channels; for the sake of facility, synthetic inductors are used in all the following experiments. A small permanent magnet of $1.3 \mathrm{gr}$ is attached to the tip of every blade and 38 of them (one every two blades) are used with custom-made voice coil actuators to excite the structure; the voice coil actuators are controlled independently with an array of 38 current amplifiers driven by a DSP board. The velocity of the blade tips is measured with a noncontact 2D Polytec laser scanner. A similar experimental setup is presented in [22].
5.2. Mistuning. The mistuning [13] is due to slight differences in the blade geometry which make some of them more flexible than others and is responsible for a nonuniform distribution of the strain energy among the blades. The mistuning destroys the rotational periodicity and, in the worst situations, introduces localization effects. The mistuned modes have no longer pure harmonic shapes (circumferentially) but include various harmonic components, and there are no more cosine and sine modes with exactly the same frequency.

The bladed drum tested in this project is a laboratory prototype which exhibits a substantial mistuning, with frequency deviations well in excess of the 1-2\% usually met in industrial bladed structures [23]. Figure 12 shows the distribution of the frequency deviation of individual blades (measured from impulse responses) of the test structure; one notices that most of the blades have a frequency deviation of the order of $2 \%$ or less, but a few of them, in the vicinity of blade \#65, have abnormally large deviations. The figure also shows two mode shapes (measured at the blade tip) which suggest the mode 1F7 with $n=7$ diameters; their natural frequencies are within $0.42 \%$; one sees that the blade mistuning alters significantly the mode shapes which are no longer harmonic 


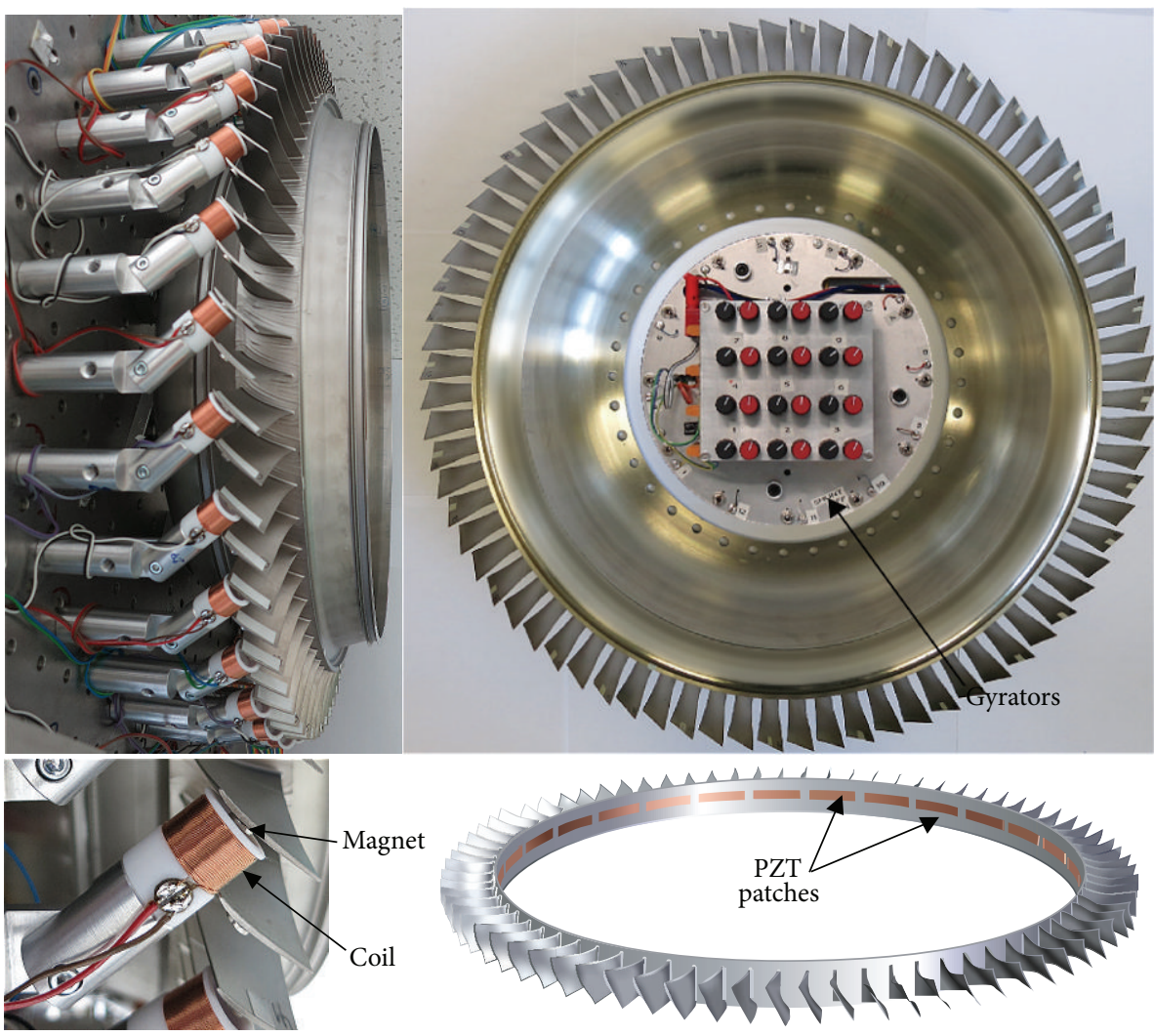

FIGURE 11: Experimental setup: bladed drum with 76 blades equipped with 28 PZT patches glued on the inner side of the drum. 28 independent gyrator circuits are used to provide synthetic inductors. 76 small permanent magnets are glued at the tip of the blades and 38 of them are used with (noncontact) voice coil actuators to provide a wide range of excitations to the structure.

in the circumferential direction; the two mode shapes have a spatial phase shift close to $90^{\circ}$ and are close to harmonic with 7 nodal diameters in the part of the structure where the blades have low frequency deviations (up to blade \#50) but are quite different where the mistuning is large. Figure 13 shows the Fourier coefficients of the observed mode shapes; one sees that the seventh harmonic is indeed the largest, but many other components exist.

5.3. Shunt Damping of Blade Mode 1F7. The mock-up is equipped with $m=N / 2=38$ voice coil actuators. The excitation may be appropriated to the mode with $n$ nodal diameters by shaping the actuator forces according to

$$
f_{k}(t)=\eta(t) \cos \left(n \theta_{k}+\phi\right), \quad \theta_{k}=\frac{4 \pi k}{N}, \quad\left(k=1, \ldots, \frac{N}{2}\right),
$$

where $\eta(t)$ is a band-limited white noise in a frequency band $\left[\omega_{1}, \omega_{2}\right]$ including all the modes of the $1 \mathrm{~F}$ family and $\phi$ is a constant angle which may be selected to maximize the response of the targeted modes.

Figure 14(a) shows the normalized FRF $\dot{x} / \eta$ between the forcing function and the velocity of blade \#19 in the frequency range of the $1 \mathrm{~F}$ family for three different conditions: (i) without shunt (all the electrodes are open), (ii) the $28 \mathrm{PZT}$ patches shunted independently on separate RL circuits using
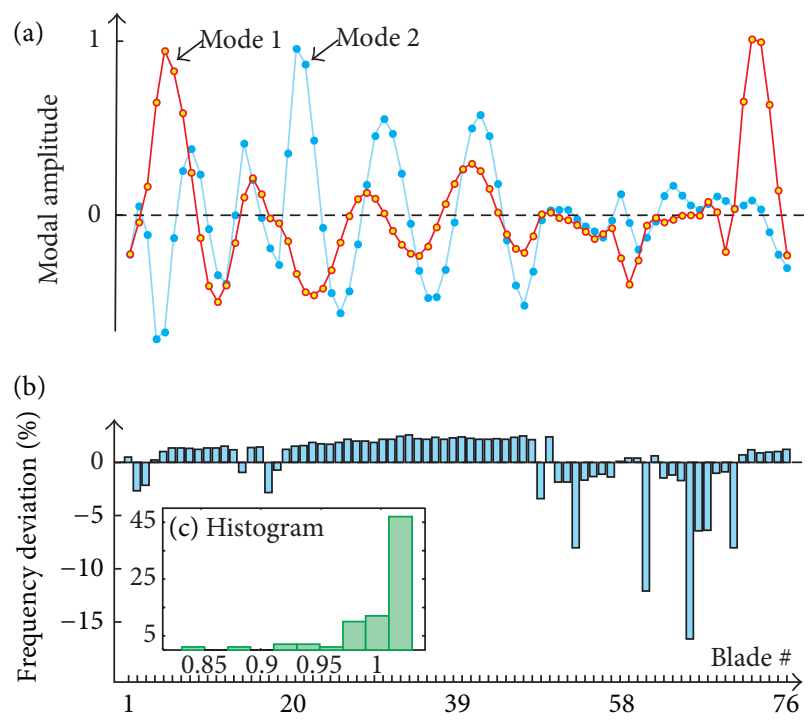

Figure 12: (a) Shapes of the two modes closer to $n=7$ diameters. (b) Distribution of the frequency deviation of the various blades (the frequencies are measured from impulse response tests); the blades in the vicinity of blade \#65 have abnormally large deviations. (c) Histogram of the frequency distribution. 

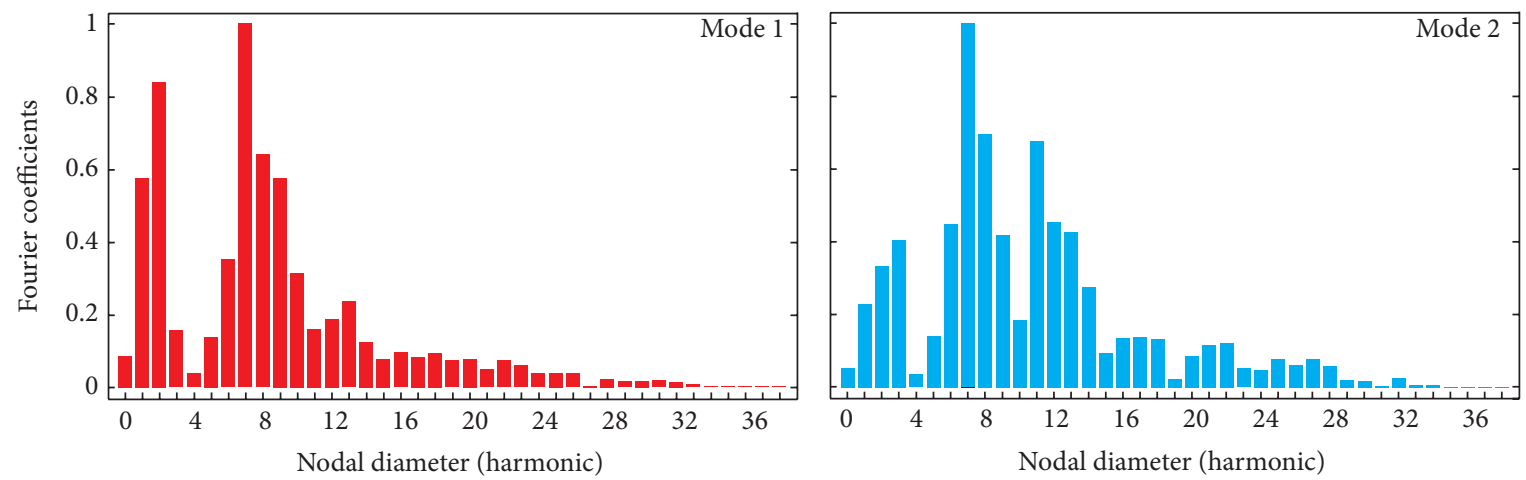

Figure 13: Distribution of the Fourier coefficients of the spatial decomposition of the two modes closer to $n=7$ diameters.

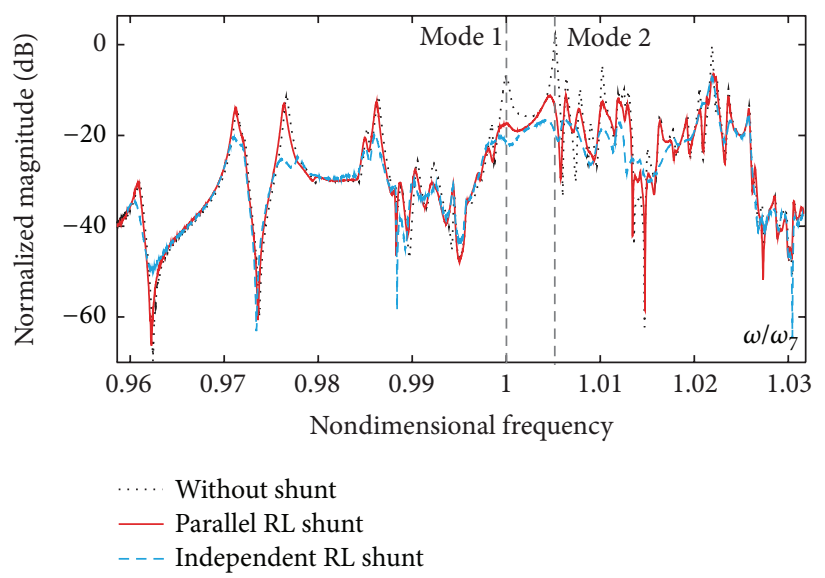

(a)

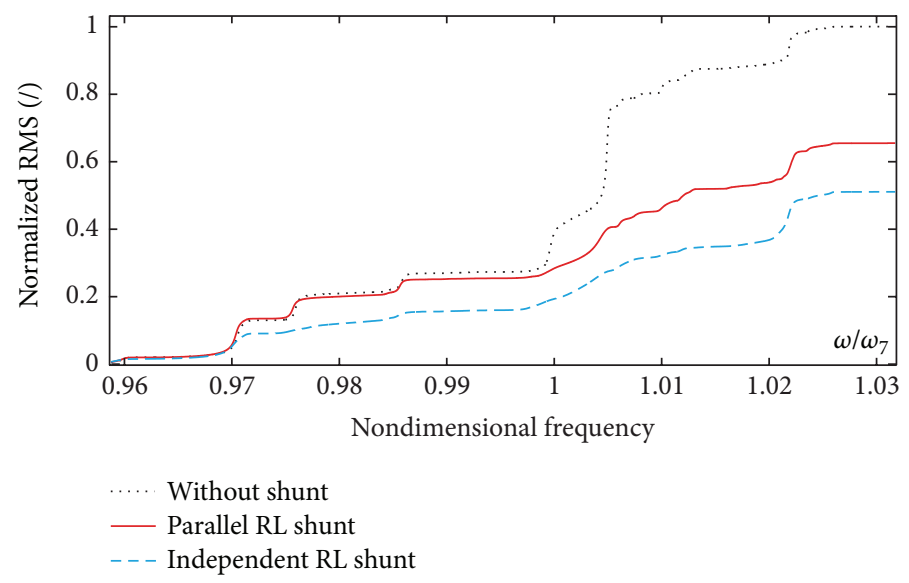

(b)

FIGURE 14: (a) Experimental normalized FRF $T(\omega)=|\dot{x} / \eta|$ between the appropriate excitation and the response of blade \#19 in the vicinity of $\omega_{7}$ for various tuning conditions of the RL shunt. (b) Cumulative RMS response $\sigma(\omega)$.

28 synthetic inductors $(L=0.7 \mathrm{H})$, and (iii) the 28 patches connected in 4 independent groups of 7 patches working in parallel (it is not possible to use 2 independent groups of 14 patches, because all the patches were mounted with the same polarization); the value of the inductance is in this case $L=100 \mathrm{mH}$. The resistance in the RL loop is set to zero because the value of the internal resistance of the synthetic inductors exceeds the value of the optimal resistance. The electrical connections of the parallel shunt are shown in the upper part of Figure 15. If the third and fourth sets of patches had been mounted with inverted polarization, the first and third sets could have been mounted in parallel and similarly for the second and fourth sets, leaving only two independent groups instead of four.

Comparing the three curves of Figure 14, one can observe that the independent shunt operates on most of the modes of the $1 \mathrm{~F}$ family (not all, because there are only 28 patches), while the parallel shunt tends to have a more limited action, mostly on mode 1 1F7 with 7 diameters. On the contrary to numerical predictions, the parallel shunt is not quite as effective as the independent shunt even for the 1F7 mode, mainly because of the presence of many additional spatial frequency components (Figure 13). Thus, the reduced effectiveness of the parallel shunt is attributed to the abnormal blade mistuning of the test article.

5.4. Shunt Damping of Drum Mode D4. The foregoing example considered blade-dominated modes with $n=7$ nodal diameters which are strongly affected by the blade mistuning; the blade mistuning of the test article is not quite representative of an industrial manufacturing process. In order to investigate the potential of the parallel shunt with moderate mistuning, the shunt damping of the drumdominated modes with $n=4$ nodal diameters has been investigated. The blade mistuning does not affect significantly the drum-dominated mode shapes (Figure 16) (it is interesting to note that blade \#60 which has a larger amplitude has a mistuning of $-12 \%$ (Figure 12), which almost coincides with the frequency of the D4 mode). However, because the drum structure is clamped at only 9 points, the sine and cosine modes do not occur at exactly the same frequency. The distribution of the Fourier coefficients of the spatial decomposition of the two modes is shown in Figure 17. The excitation (14) is appropriated to the modes with $n=4$ nodal diameters. The configuration with 28 PZT patches is not particularly appropriate for a mode with $n=4$ 

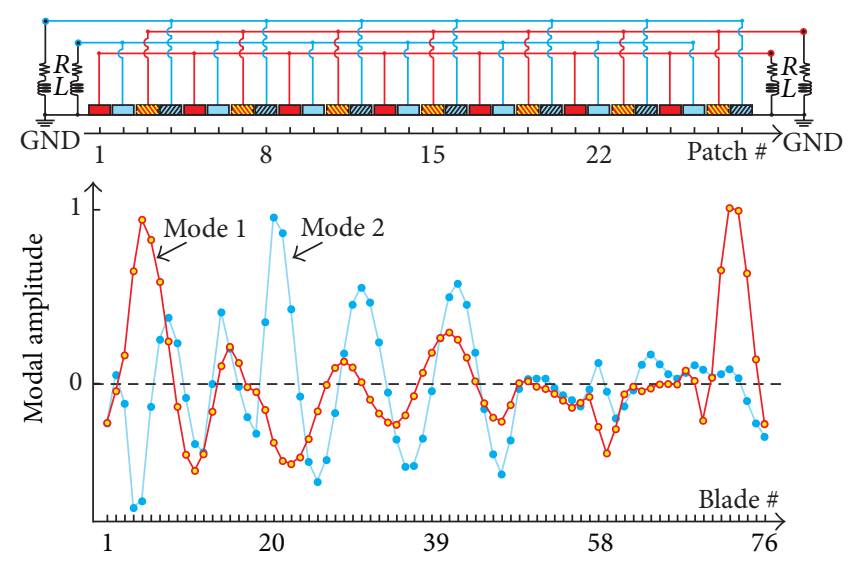

FIGURE 15: Numbering of the 28 PZT patches and numbering of the blades along the circumference of the drum. The upper figure indicates the electrical connections of the PZT patches in 4 independent loops for the parallel shunt; the lower figure shows the shape of the targeted modes (1F7).
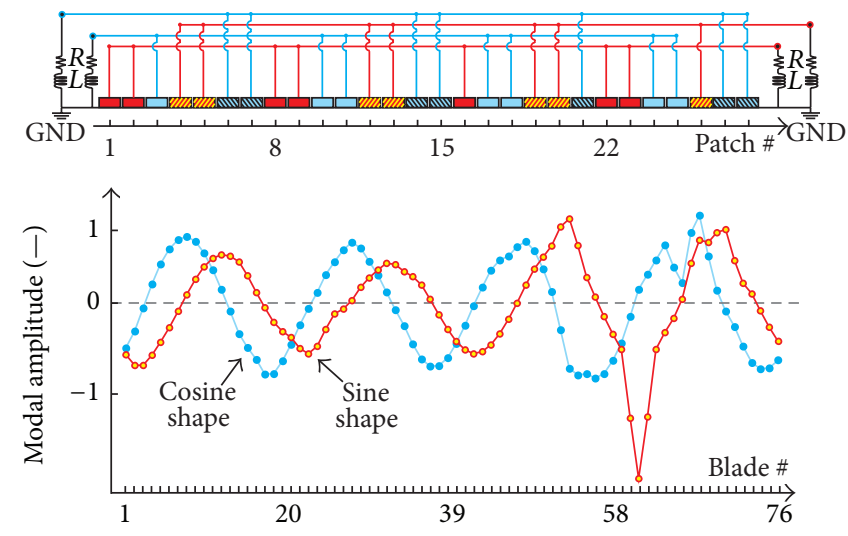

FIGURE 16: Below: shape of the drum-dominated mode D4 with $n=4$ nodal diameters (sine and cosine modes). Above: electrical connections of the PZT patches in 4 independent loops for the parallel shunt (all PZT patches of the same color are connected in parallel).

diameters (28 is not a multiple of $4 n$ ) and the organization of the four parallel loops has been based on the examination of the mode shapes; it is shown in the upper part of Figure 16.

Figure 18(a) compares the normalized FRF $\dot{x} / \eta$ between the forcing function and the velocity of blade \#52 for three different conditions: (i) without shunt (all the electrodes are open), (ii) the 28 PZT patches shunted independently on separate RL circuits using 28 synthetic inductors $(L \simeq 1 \mathrm{H})$, and (iii) the 28 patches connected in 4 independent groups of 7 patches working in parallel, with the electrical connections indicated in Figure 16; the four synthetic inductors have in this case $L=140 \mathrm{mH}$. Here again, the resistance in the RL loop is set to zero because the value of the internal resistance of the synthetic inductors exceeds the value of the optimal resistance. The cumulative RMS response $\sigma(\omega)$ for the three electrical connections is shown in Figure 18(b). One sees that, in this case where the mode shapes are much closer to circumferentially harmonic functions, the performance of the parallel shunt is nearly the same as that of the independent shunt for the targeted modes.

\section{Conclusion}

This paper considers the RL shunt damping of rotationally periodic structures with an array of regularly spaced piezoelectric patches. For the bladed drum considered in this study, it is possible to achieve a significant reduction of the blade vibrations with an array of piezoelectric transducers organized on the inner side of the blade support rim, without any disturbance to the compressor flow. The use of independent loops for the various transducers allows damping the entire $1 \mathrm{~F}$ family of modes, but it usually requires synthetic inductors which are not practical for rotating machines. However, in many applications, by superimposing the "zigzag" lines on the natural frequency versus nodal diameters plot, it is possible to identify the critical mode with $n$ nodal diameters which must be targeted for damping. If this is the case, by organizing $4 n$ piezoelectric transducers (PZT patches) in two parallel loops of $2 n$ patches each, the demand on the inductors may be reduced by $4 n^{2}$ as compared to independent loops, allowing the use of passive components. The method has been tested on a circular plate and on 

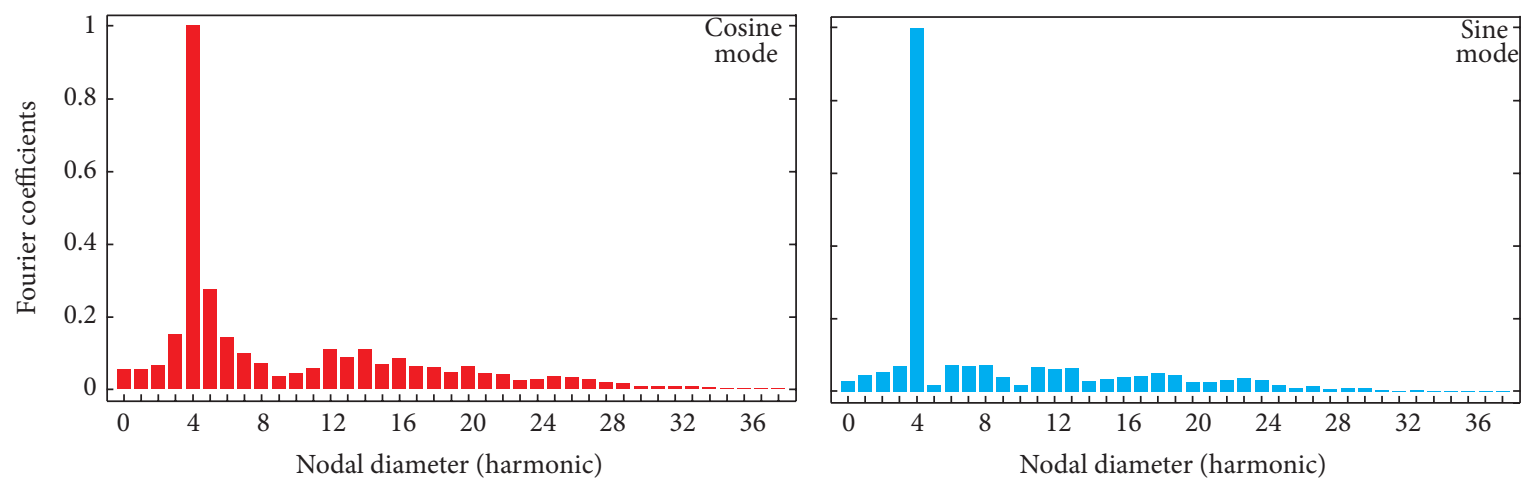

Figure 17: (a) Distribution of the Fourier coefficients of the spatial decomposition of the sine and cosine drum-dominated modes D4 with $n=4$ diameters.
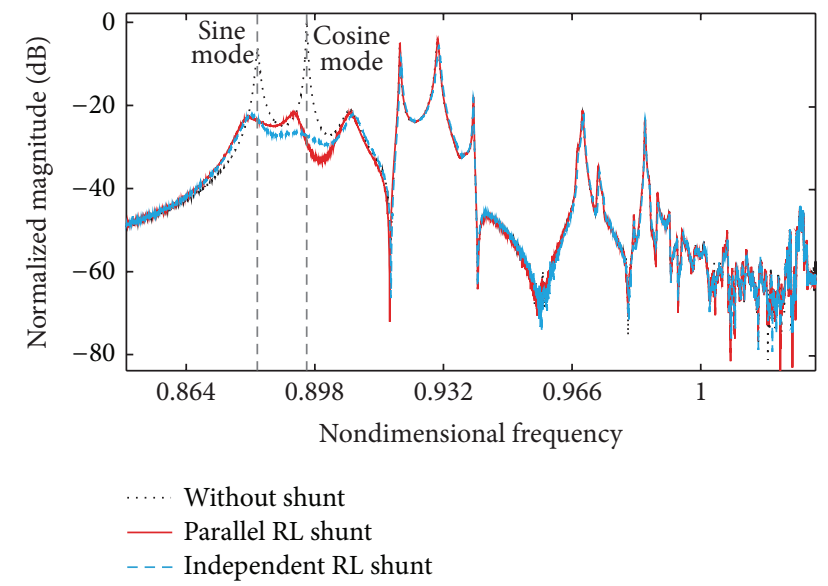

(a)

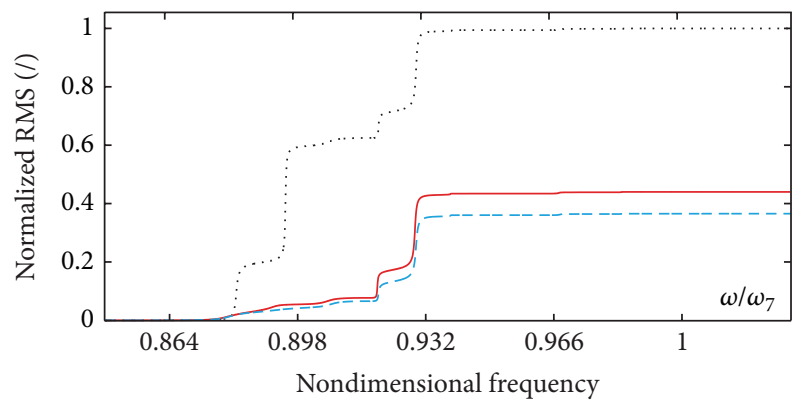

Without shunt

- Parallel RL shunt

- - - Independent RL shunt

(b)

FIGURE 18: (a) Experimental normalized FRF $T(\omega)=|\dot{x} / \eta|$ between the appropriate excitation and the response of blade \#52 for various tuning conditions of the RL shunt. (b) Cumulative RMS response $\sigma(\omega)$.

a bladed drum with 76 blades. It has been found very effective when the targeted mode shape is close to harmonic circumferentially. The method seems to be applicable even in the presence of a moderate blade mistuning, but the performances are significantly degraded in the presence of substantial mistuning. Further investigations on the influence of mistuning are underway.

\section{Conflict of Interests}

The authors declare that there is no conflict of interests regarding the publication of this paper.

\section{Acknowledgment}

This research was funded by "Skywin" program of Région Wallonne, project $\mathrm{HM}+$.

\section{References}

[1] J. H. Griffin, "A review of friction damping of turbine blade vibration," International Journal of Turbo and Jet Engines, vol. 7, no. 3-4, pp. 297-307, 1990.

[2] D. Laxalde, F. Thouverez, and J.-P. Lombard, "Forced response analysis of integrally bladed disks with friction ring dampers," Journal of Vibration and Acoustics, vol. 132, no. 1, Article ID 011013, 2010.

[3] J. Tang and K. W. Wang, "Vibration control of rotationally periodic structures using passive piezoelectric shunt networks and active compensation," Transactions of the ASME-Journal of Vibration and Acoustics, vol. 121, no. 3, pp. 379-390, 1999.

[4] J. L. Kauffman and G. A. Lesieutre, "Piezoelectric-based vibration reduction of turbomachinery bladed disks via resonance frequency detuning," AIAA Journal, vol. 50, no. 5, pp. 1137-1144, 2012.

[5] R. L. Forward, "Electronic damping of vibrations in optical structures," Applied Optics, vol. 18, no. 5, pp. 690-697, 1979.

[6] R. L. Forward, "Electronic damping of orthogonal bending modes in a cylindrical mast experiment," AIAA Journal of Spacecraft, vol. 18, no. 1, pp. 11-17, 1981.

[7] N. W. Hagood and A. von Flotow, "Damping of structural vibrations with piezoelectric materials and passive electrical networks," Journal of Sound and Vibration, vol. 146, no. 2, pp. 243-268, 1991.

[8] M. V. Kozlowski, D. G. Cole, and R. L. Clark, "A comprehensive study of the RL series resonant shunted piezoelectric: a feedback controls perspective," Journal of Vibration and Acoustics, vol. 133, no. 1, Article ID 011012, 2011. 
[9] J. J. Hollkamp, "Multimodal passive vibration suppression with piezoelectric materials and resonant shunts," Journal of Intelligent Material Systems and Structures, vol. 5, no. 1, pp. 49$57,1994$.

[10] D. L. Edberg, A. S. Bicos, and J. S. Fechter, "On piezoelectric energy conversion for electronic passive damping enhancement," in Proceedings of the 1991 Damping, San Diego, Calif, USA, January 1991.

[11] D. Guyomar and C. Richard, "Non-linear and hysteretic processing of piezoelement: application to vibration control, wave control and energy harvesting," International Journal of Applied Electromagnetics and Mechanics, vol. 21, no. 3-4, pp. 193-207, 2005.

[12] S. J. Wildheim, "Excitation of rotationally periodic structures," Transactions ASME-Journal of Applied Mechanics, vol. 46, no. 4, pp. 878-882, 1979.

[13] M. P. Castanier and C. Pierre, "Modeling and analysis of mistuned bladed disk vibration: status and emerging directions," Journal of Propulsion and Power, vol. 22, no. 2, pp. 384-396, 2006.

[14] D. L. Thomas, "Dynamics of rotationally periodic structures," International Journal for Numerical Methods in Engineering, vol. 14, no. 1, pp. 81-102, 1979.

[15] C. Pierre, "Mode localization and eigenvalue loci veering phenomena in disordered structures," Journal of Sound and Vibration, vol. 126, no. 3, pp. 485-502, 1988.

[16] A. Preumont, Mechatronics: Dynamics of Electromechanical and Piezoelectric Systems, Springer, 2006.

[17] B. Mokrani, Piezoelectric shunt damping of rotationally periodic structures [Ph.D. thesis], Active Structures Laboratory, Université libre de Bruxelles, Brussels, Belgium, 2015, http://scmero .ulb.ac.be/publications.php.

[18] R. Bastaits, B. Mokrani, R. Viguié, and A. Preumont, "Vibration damping of turbomachinery components," in New Trends in Smart Technologies, C. Boller and H. Janocha, Eds., Frauhofer, 2013.

[19] V. Piefort, Finite element modeling of piezoelectric active structures [Ph.D. thesis], Université Libre de Bruxelles, Active Structures Laboratory, 2001.

[20] V. Piefort, N. Loix, and A. Preumont, "Modeling of piezolaminated composite shells for vibration control," in Proceedings of the ESA Conference on Spacecraft Structures, Materials and Mechanical Testing, ESA SP-428, Braunschweig, Germany, November 1998.

[21] A. Antoniou, "Realization of gyrators using operational amplifiers, and their use in RC-active-network synthesis," Proceedings of the Institution of Electrical Engineers, vol. 116, no. 11, pp. 1838$1850,1969$.

[22] C. M. Firrone and T. Berruti, "An electromagnetic system for the noncontact excitation of bladed disks," Experimental Mechanics, vol. 52, no. 5, pp. 447-459, 2012.

[23] B.-K. Choi, J. Lentz, A. J. Rivas-Guerra, and M. P. Mignolet, "Optimization of intentional mistuning patterns for the reduction of the forced response effects of unintentional mistuning: formulation and assessment," Journal of Engineering for Gas Turbines and Power, vol. 125, no. 1, pp. 131-140, 2003. 

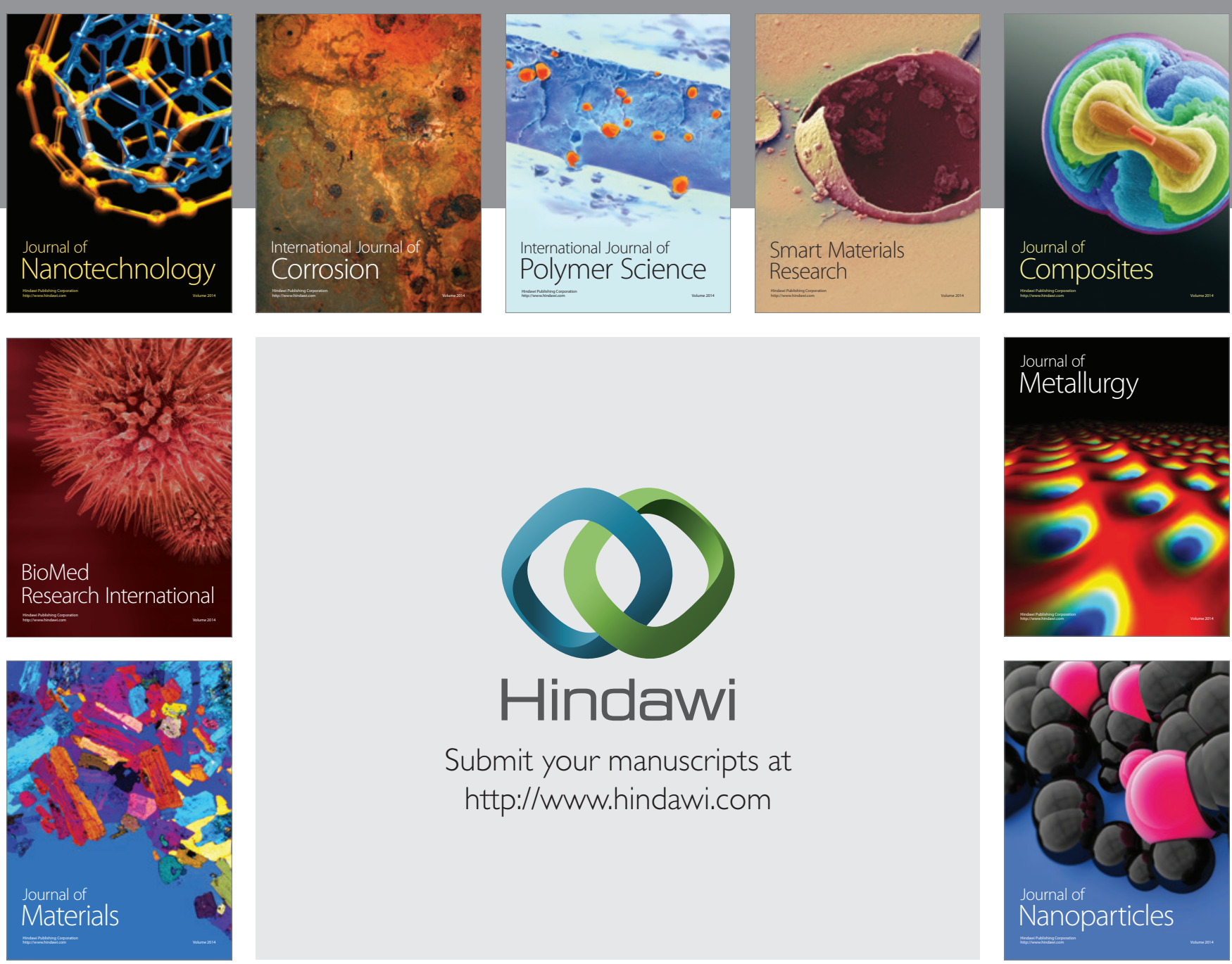

Submit your manuscripts at http://www.hindawi.com
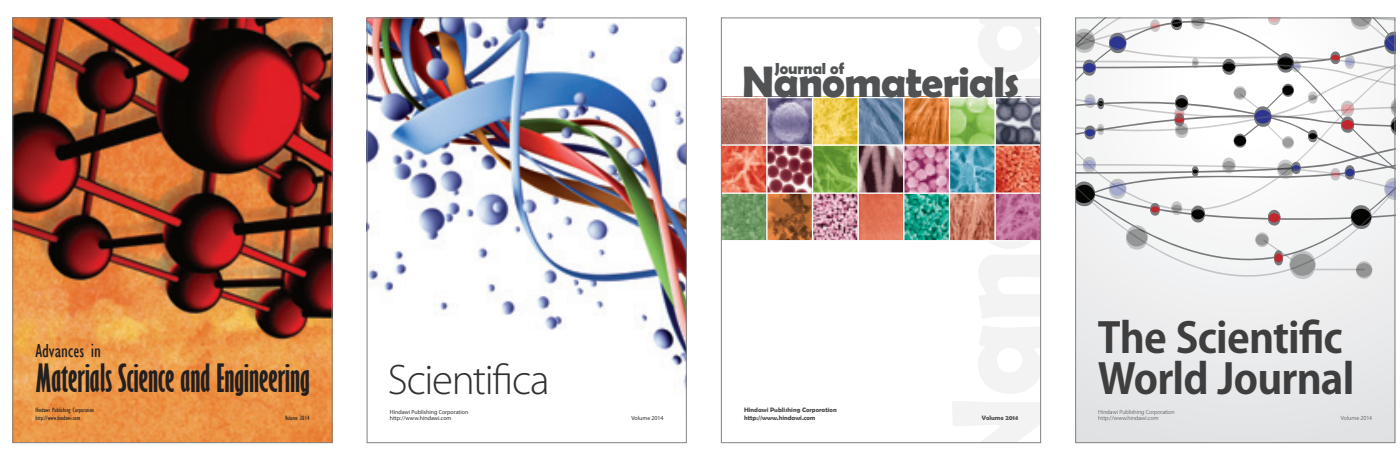

\section{The Scientific World Journal}
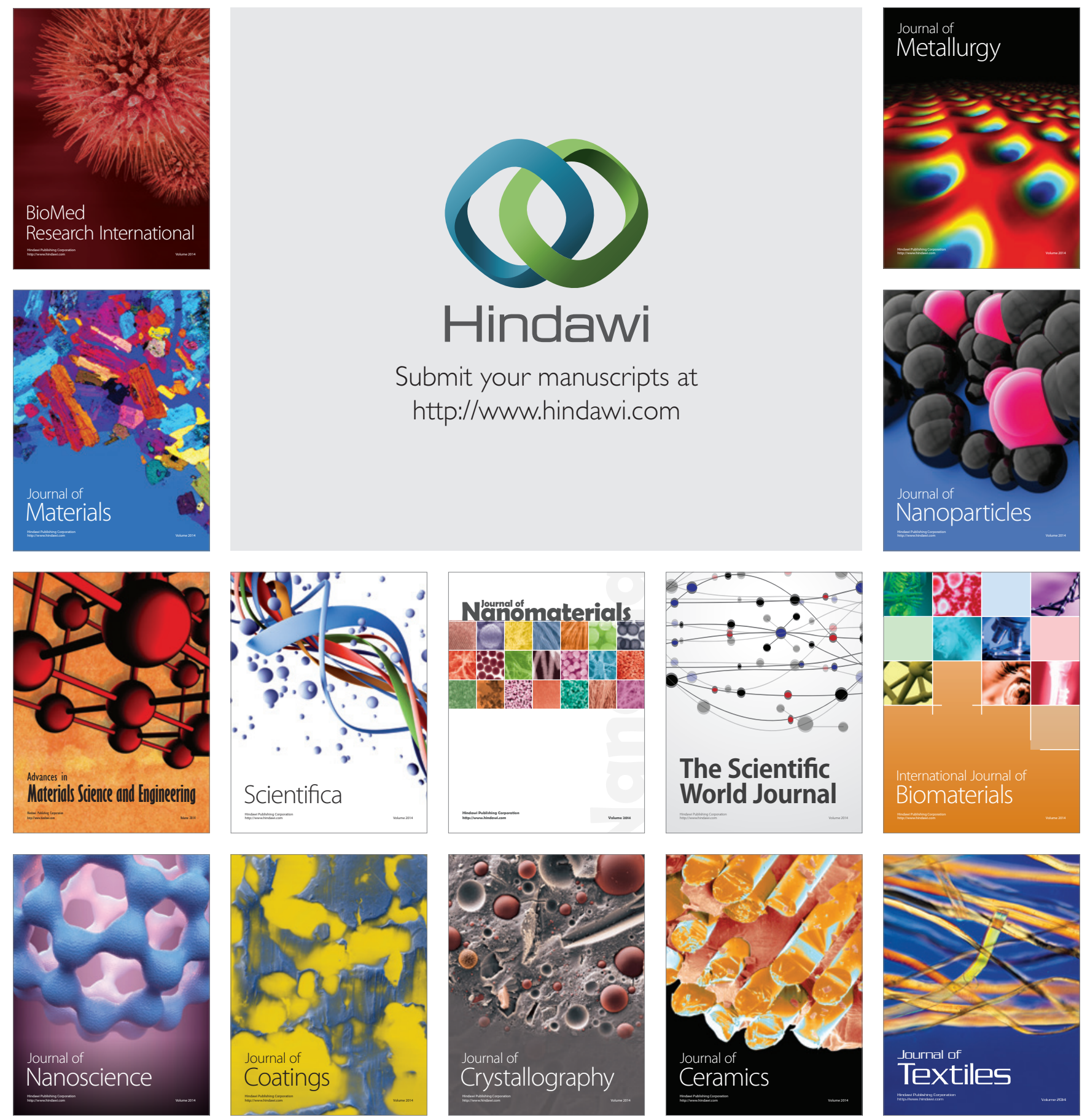